\title{
PLXND1 Gene
}

National Cancer Institute

\section{Source}

National Cancer Institute. PLXND1 Gene. NCI Thesaurus. Code C114410.

This gene is involved in semaphorin binding and signaling. 